\title{
Organometallic benzene-vanadium wire: A one-dimensional half-metallic ferromagnet
}

\author{
Maslyuk, V.; Bagrets, A.; Meded, V.; Arnold, A.; Evers, F.; Brandbyge, Mads; Bredow, T.; Mertig, I.
}

Published in:

Physical Review Letters

Link to article, DOI:

10.1103/PhysRevLett.97.097201

Publication date:

2006

Document Version

Publisher's PDF, also known as Version of record

Link back to DTU Orbit

Citation (APA):

Maslyuk, V., Bagrets, A., Meded, V., Arnold, A., Evers, F., Brandbyge, M., Bredow, T., \& Mertig, I. (2006).

Organometallic benzene-vanadium wire: A one-dimensional half-metallic ferromagnet. Physical Review Letters, 97(9), 101103. https://doi.org/10.1103/PhysRevLett.97.097201

\section{General rights}

Copyright and moral rights for the publications made accessible in the public portal are retained by the authors and/or other copyright owners and it is a condition of accessing publications that users recognise and abide by the legal requirements associated with these rights.

- Users may download and print one copy of any publication from the public portal for the purpose of private study or research.

- You may not further distribute the material or use it for any profit-making activity or commercial gain

- You may freely distribute the URL identifying the publication in the public portal 


\title{
Organometallic Benzene-Vanadium Wire: A One-Dimensional Half-Metallic Ferromagnet
}

\author{
Volodymyr V. Maslyuk, ${ }^{1}$ Alexei Bagrets, ${ }^{2,1}$ Velimir Meded, ${ }^{2,3}$ Andreas Arnold, ${ }^{2}$ Ferdinand Evers, ${ }^{2,4}$ Mads Brandbyge, ${ }^{5}$ \\ Thomas Bredow, ${ }^{6}$ and Ingrid Mertig ${ }^{1}$ \\ ${ }^{1}$ Martin-Luther-Universität Halle-Wittenberg, Fachbereich Physik, D-06099 Halle, Germany \\ ${ }^{2}$ Institut für Nanotechnologie, Forschungszentrum Karlsruhe, D-76344, Germany \\ ${ }^{3}$ Condensed Matter Theory Group, Department of Physics, Uppsala University, SE-751, Sweden \\ ${ }^{4}$ Institut für Theorie der Kondensierten Materie, Universität Karlsruhe, 76128 Karlsruhe, Germany \\ ${ }^{5}$ NanoDTU, MIC-Department of Micro and Nanotechnology, Technical University of Denmark, DK-2800 Lyngby, Denmark \\ ${ }^{6}$ Institut für Theoretische Chemie, Universität Hannover, D-30167 Hannover, Germany
}

(Received 5 October 2005; published 30 August 2006)

\begin{abstract}
Using density functional theory we perform theoretical investigations of the electronic properties of a freestanding one-dimensional organometallic vanadium-benzene wire. This system represents the limiting case of multidecker $\mathrm{V}_{n}\left(\mathrm{C}_{6} \mathrm{H}_{6}\right)_{n+1}$ clusters which can be synthesized with established methods. We predict that the ground state of the wire is a $100 \%$ spin-polarized ferromagnet (half-metal). Its density of states is metallic at the Fermi energy for the minority electrons and shows a semiconductor gap for the majority electrons. We find that the half-metallic behavior is conserved up to $12 \%$ longitudinal elongation of the wire. Ab initio electron transport calculations reveal that finite size vanadium-benzene clusters coupled to ferromagnetic $\mathrm{Ni}$ or Co electrodes will work as nearly perfect spin filters.
\end{abstract}

DOI: 10.1103/PhysRevLett.97.097201

During the last few years, molecular magnets have been attracting enormous attention because they are considered as potential candidates for future applications in highdensity information storage and quantum computing. Among such novel systems, we would like to focus on vanadium-benzene multidecker clusters $\left(\mathrm{V}_{n} \mathrm{Bz}_{n+1}, \mathrm{Bz}=\right.$ $\mathrm{C}_{6} \mathrm{H}_{6}$ ). These are molecular magnets appearing in a gas phase [1,2]. The synthesis of the $\mathrm{V}$-Bz clusters was realized up to $n=6$ during a reaction of laser-vaporized metal atoms with benzene in a $\mathrm{He}$ atmosphere [2,3]. The mass spectrometric measurements $[2,4]$ and theoretical studies [5-7] suggested that $\mathrm{V}_{n} \mathrm{Bz}_{n+1}$ clusters should have a onedimensional structure which is settled by the metal-ligand interactions confined to a single molecular axis.

In this Letter, we present an $a b$ initio study of the infinite one-dimensional (1D) organometallic vanadium-benzene (V-Bz) wire shown in Fig. 1 which could be considered as the limiting case of the $\mathrm{V}_{n} \mathrm{Bz}_{n+1}$ clusters with $n \rightarrow \infty$. We have performed calculations of the electronic structure of the system and predict that the wire under consideration is a new, unusual example of a one-dimensional half-metallic ferromagnet. It has an integer magnetic moment of $1 \mu_{B}$ per unit cell and a spin-polarized band structure with a finite density of states at the Fermi level for one spin channel and a semiconductor gap for the other (Fig. 2). Thus, the electronic properties of this organometallic wire are similar to that of the known Heusler compound, $\mathrm{NiMnSb}$, which has been predicted to be a half-metal by de Groot et al. in 1983 [8]. Furthermore, our electron transport calculations have shown that a finite fragment of the wire would act as a nearly perfect molecular spin filter (Fig. 4) when a V-Bz cluster is placed between magnetic electrodes [9].
PACS numbers: 75.50.Pp, 71.20.-b, 75.75.+a, 85.75.-d

The calculations of the properties of the $\mathrm{V}-\mathrm{Bz}$ wire are based on density functional theory and were performed within the linear combination of atomic orbitals formalism implemented in the highly accurate CRYSTAL code [10]. The Perdew-Wang [11] generalized-gradient approximation (GGA) for the exchange and correlation energy was used. The description of the electron subsystem was done with the full electron basis set 6-311G** [12] for carbon and hydrogen atoms and with a frozen core basis set SBKJC [13] for vanadium. Convergent results were achieved using the Pack-Monkhorst grid with $24 k$ points in the 1D Brillouin zone. The wire under consideration (Fig. 1) has a one-dimensional hexagonal lattice with

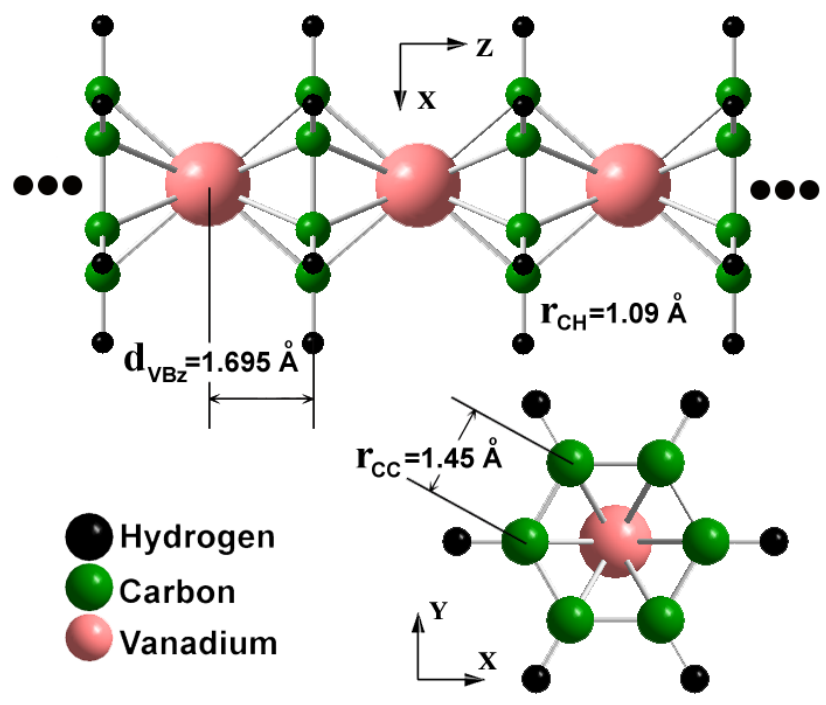

FIG. 1 (color online). The structure of the $\mathrm{V}\left(\mathrm{C}_{6} \mathrm{H}_{6}\right)$ wire. 

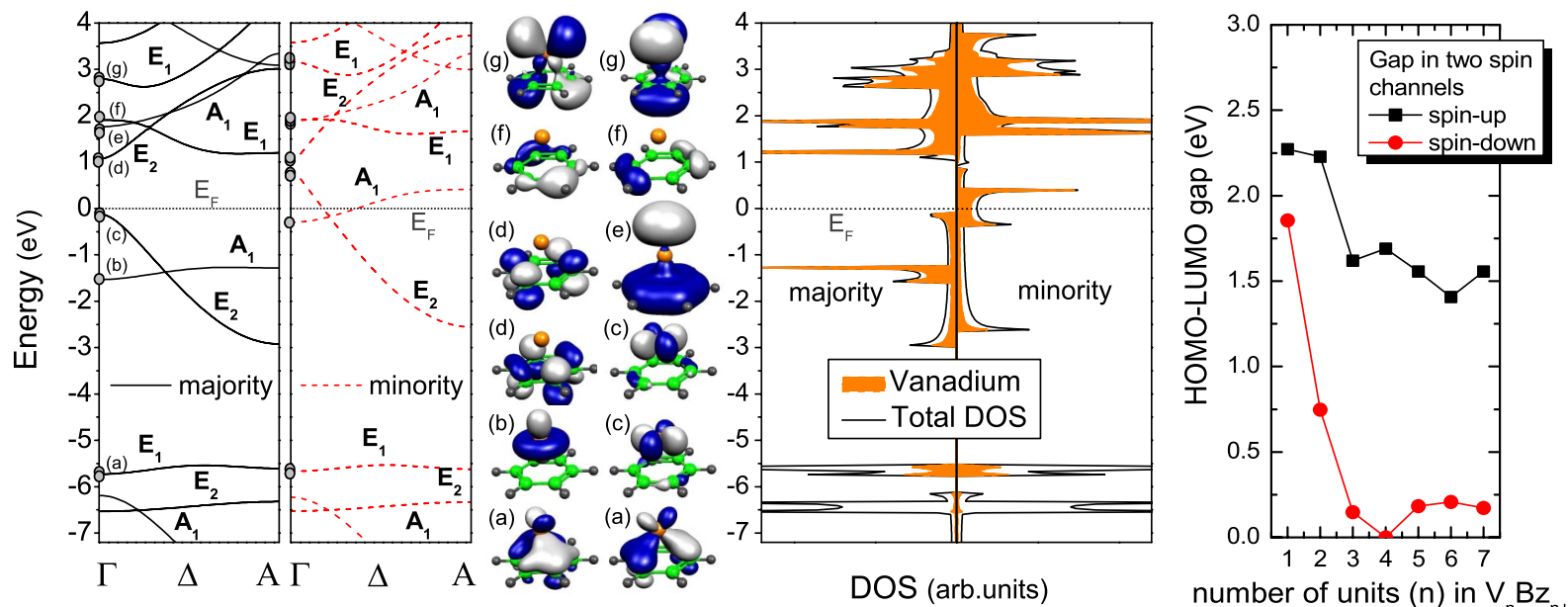

FIG. 2 (color online). The spin-resolved band structure (left plot) and the DOS (plot in the middle) of the $\mathrm{V}\left(\mathrm{C}_{6} \mathrm{H}_{6}\right)$ wire in the ferromagnetic phase. The labels at the band structure refer to crystalline orbitals of the wire calculated for the $\Gamma$ point. These functions are shown in the center. The corresponding majority and minority spin orbitals have practically the same shape. The right panel shows values of the HOMO-LUMO gap in two spin channels for $\mathrm{V}_{n}\left(\mathrm{C}_{6} \mathrm{H}_{6}\right)_{n+1}$ clusters as a function of $n$.

$P 6 / m m m\left(D_{6 h}^{1}\right)$ rod symmetry. From calculations of the total energy of the system we have found that the optimal structure corresponds to the following interatomic distances: $d_{\mathrm{VBz}}=1.69 \AA, r_{\mathrm{CC}}=1.45 \AA$, and $r_{\mathrm{CH}}=1.09 \AA$, that is in good agreement with other studies $[14,15]$. We have also examined another, staggered-type, geometry of the wire when the consecutive benzol rings were rotated by $30^{\circ}$ with respect to each other. We have found that the staggered geometry is energetically less favorable, with a total energy per unit cell being $0.06 \mathrm{eV}$ higher than the lowest-energy of the wire with $D_{6 h}^{1}$ symmetry. Moreover, our calculations showed that the rotation of the benzene ring did not change the electronic properties of the wire.

In Fig. 2 we present the spin-polarized band structure of the V-Bz wire (left) [16] together with the corresponding density of states (middle). We accompanied these data by plotting the crystalline orbitals evaluated at the $\Gamma$ point. The symmetry of the bands corresponds to the $C_{6 v}$ point group relevant for the Bloch vectors. We see that for the minority spin electrons the $A_{1}$ band and the doubly degenerate $E_{2}$ band cross the Fermi level, while for the majority spin electrons the Fermi energy $\left(E_{F}\right)$ lies in the gap. The direct gap has a width of $E_{g}=1.18 \mathrm{eV}$ while the spin-flip gap is $\Delta_{s}=0.12 \mathrm{eV}$ at the $\Gamma$ point. Such a system would have ballistic conductance of $3 e^{2} / h$ in one spin channel and zero transmission for the other. To verify the validity of the obtained result with respect to a more careful treatment of the on-site correlation effects between $d$ electrons of vanadium, we have performed additional calculations within the local density approximation LDA $+U$ scheme [17] using the projector augmented wave method [18] implemented in the VASP [19], as well as within the hybrid B3LYP exchange-correlation functional [20]. It was confirmed that the half-metallic behavior is robust against details of the calculations. The size of the gap and the relative positions of bands are of course subject to the chosen method (Table I). The inclusion of a Hubbard $U$ term results in a downward shift of the majority $A_{1}$ and $E_{2}$ bands leading to an increase of the spin-flip gap $\Delta_{s}$, while the minority bands crossing the Fermi level are unaffected. The B3LYP functional pulls the majority spin bands even more strongly away from $E_{F}$. In addition, the antiferromagnetic state of the wire was found to be energetically less preferable as compared with the ferromagnetic configuration.

The spin-density map (Fig. 3) indicates a small negative magnetic moment $\left(-0.28 \mu_{B}\right)$ redistributed over $6 \mathrm{C}$ atoms and a quite localized positive magnetic moment at the $\mathrm{V}$ atom $\left(+1.28 \mu_{B}\right)$. The integer magnetic moment $\left(1.0 \mu_{B}\right)$ of the wire unit cell arises mainly from the minority spin $A_{1}$ states labeled as (b) in Fig. 2 that is in agreement with theoretical [6] and experimental [1,2,21-23] data. Since the corresponding wave function (b) is nearly a pure $d_{3 z^{2}-r^{2}}$ vanadium orbital, its symmetry is reflected in the angular distribution of the spin density around the $\mathrm{V}$ atom. Furthermore, we note that the small negative magnetic moment on the $\mathrm{C}$ atoms results from the uncompensated $E_{2}$ band (c) whose molecular orbital contains an admixture of the carbon $p$ states (Fig. 2).

TABLE I. The spin-flip gap $\Delta_{s}$ (the energy difference between $E_{F}$ and the top of the majority spin valence band), the direct gap $E_{g}$, and the lattice constant of the $\mathrm{V}\left(\mathrm{C}_{6} \mathrm{H}_{6}\right)$ wire.

\begin{tabular}{|c|c|c|c|c|}
\hline & Method & $\Delta_{s}, \mathrm{eV}$ & $E_{g}, \mathrm{eV}$ & $d_{V B z}, \AA$ \\
\hline GGA & & 0.12 & 1.18 & 1.695 \\
\hline $\mathrm{LDA}+U(2 \mathrm{eV})$ & & 0.22 & 1.04 & 1.663 \\
\hline $\mathrm{LDA}+U(3 \mathrm{eV})$ & & 0.57 & 1.25 & 1.676 \\
\hline $\mathrm{LDA}+U(4 \mathrm{eV})$ & & 1.04 & 1.58 & 1.696 \\
\hline B3LYP & & 1.22 & 2.52 & 1.760 \\
\hline
\end{tabular}




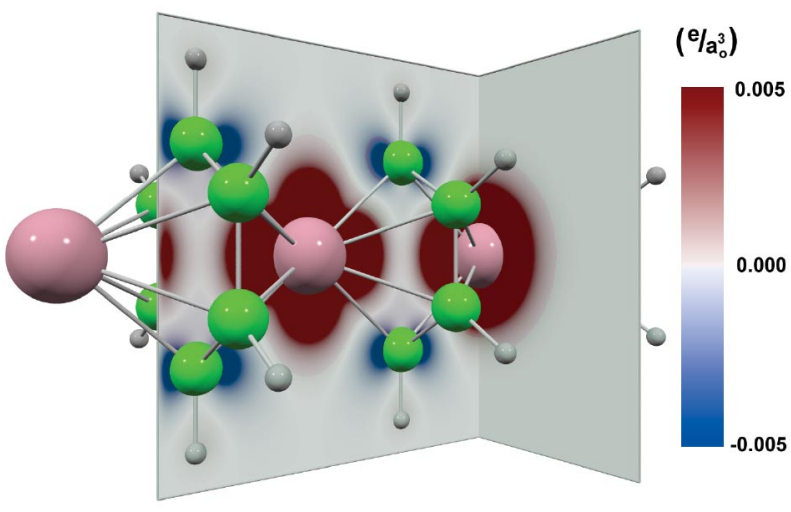

FIG. 3 (color online). Spin-density map for the $\mathrm{V}\left(\mathrm{C}_{6} \mathrm{H}_{6}\right)$ wire. Local magnetic moments are $1.28 \mu_{B}$ at $\mathrm{V}$ atom, and $-0.047 \mu_{B}$ at each $\mathrm{C}$ atom.

The analysis of the data shown in Fig. 2 leads to the following picture of the formation of the ground state. The wire bands being responsible for the magnetism lie above $-6 \mathrm{eV}$ with respect to $E_{F}$ and are formed by the hybridized states of vanadium and benzene. The strong crystalline field splits the vanadium $3 d$ levels to a singlet $A_{1}$ $\left(d_{3 z^{2}-r^{2}}\right)$ and two doublets, of $E_{1}\left(d_{x z}, d_{y z}\right)$ and $E_{2}\left(d_{x y}\right.$, $\left.d_{x^{2}-y^{2}}\right)$ symmetry. The doubly degenerate $E_{1}$ band just above $-6 \mathrm{eV}$ is formed mainly by the HOMO and the HOMO - $1 \pi$-type orbitals of benzene with an admixture of the $d_{x z}, d_{y z}$ orbitals of vanadium. Because of the strong hybridization effect between the states of the same symmetry, the vanadium $d_{x z}, d_{y z}$ levels are pushed well above the Fermi energy where they are coupled with antibonding benzene states leading to the formation of the two different bands of $E_{1}$ symmetry, marked by (f) and (g). The remaining $d_{3 z^{2}-r^{2}}$ and $d_{x y}, d_{x^{2}-y^{2}}$ vanadium states form two spinsplit bands [(b) and (c)] nearby the Fermi level, of $A_{1}$ and $E_{2}$ symmetry, respectively. The isolated $\mathrm{V}$ atom has [Ar] $3 d^{3} 4 s^{2}$ electronic configuration. However, in the wire the vanadium $s$ levels are shifted above $E_{F}$ and take part in the formation of the $A_{1}$ band labeled as (e). Thus five electrons in total wish to occupy three levels $\left(A_{1}\right.$ and $\left.E_{2}\right)$, which are available per spin. The majority spin electrons fill completely two bands of $A_{1}$ and $E_{2}$ symmetry, which therefore are placed below $E_{F}$. The remaining two electrons of each unit cell occupy partially the minority spin $A_{1}$ and $E_{2}$ bands. Consequently, both of them are crossing the Fermi level. To conclude, the magnetic moment per unit cell is forced to be $1.0 \mu_{B}$.

The unusual electronic properties of the infinite 1D wire remain in molecules of finite length $(n \leq 6)$. We now show how these molecular wires, which have already been synthesized [2,3], act as highly effective spin filters. That is already seen from results presented in the right panel of Fig. 2 where one can notice that for clusters with $n \geq 3$ the HOMO-LUMO gap $(\sim 0.2 \mathrm{eV})$ between discrete molecular levels in the minority spin channel is 1 order of magnitude smaller than the gap in the majority spin channel $(\sim 1.5 \mathrm{eV})$. We have rigorously verified the idea by per- forming two sets of independent fully $a b$ initio transport calculations through the $\mathrm{V}-\mathrm{Bz}$ clusters. Molecules were coupled to $\mathrm{Co}$ and $\mathrm{Ni}$ fcc (001) electrodes with frontier benzene rings placed on top of the hollow sites of the fcc (001) surface that corresponds to the energetically most stable configuration [24]. Examples of the transmission spectra $T(E)$ for a cluster with $n=3$ units are shown in Fig. 4. We employed TRANSIESTA [25] in the case of a molecule suspended between Co leads (dashed lines). In the case of Ni electrodes [26] (solid lines), we used the TURBOMOLE package [27] supplemented by absorbing boundary conditions for the self-energy [28]. The insulating gap is conserved in the spin-up transmission. In the linear regime, the spin polarization of the electron current $\left[T_{\downarrow}\left(E_{F}\right)-T_{\uparrow}\left(E_{F}\right)\right] / T_{\downarrow}\left(E_{F}\right)$ approaches $90 \%$. The different types of conduction mechanisms in the two spin channels (tunneling versus metallic) are reflected in the spaceresolved density of states (DOS) at $E_{F}$ shown in the insets of Fig. 4.

Turning back to the infinite V-Bz wire, we can ask the following question: what is the mechanism of the exchange interaction between localized magnetic moments of the $\mathrm{V}$ atoms? Possible answers are: (i) direct exchange, and (ii) indirect, superexchange interaction which can be established through the $\pi$-type orbitals of benzene. Pursuing the first mechanism, we have compared spin-projected local densities of states (LDOS) at the V atom for the ferromagnetic (FM) and the antiferromagnetic (AFM) state and found the LDOS not to be identical. That indicates the direct exchange, since without mutual influence of the localized moments one could expect the LDOS for both phases to be almost alike. We observed that the AFM phase was not any more half-metallic and the vanadium magnetic moment was reduced to $0.62 \mu_{B}$. The direct exchange inter-

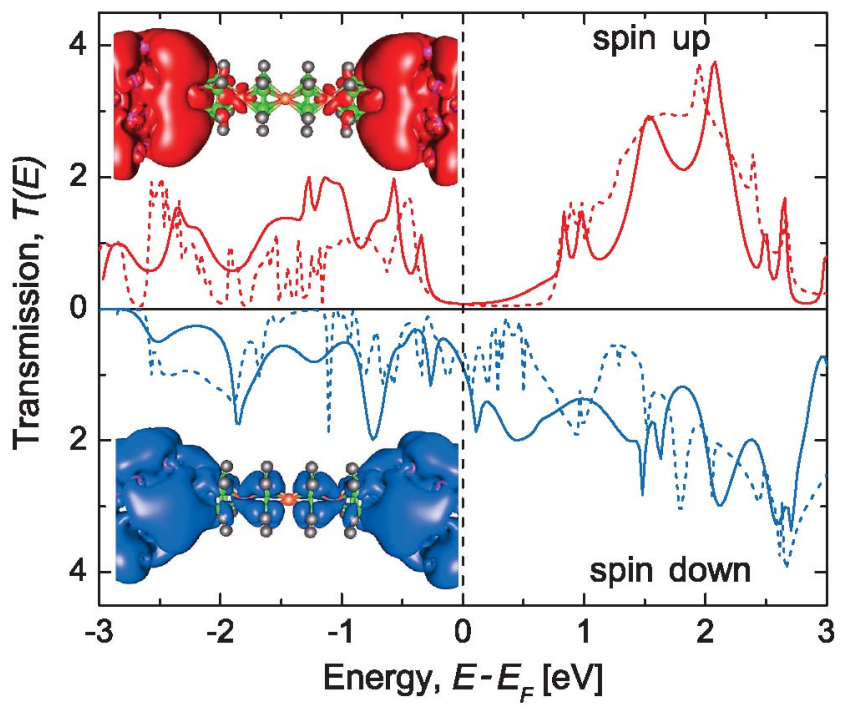

FIG. 4 (color online). Transmission spectra of the $\mathrm{V}_{3} \mathrm{Bz}_{4}$ cluster suspended between either Co (dashed line) or Ni (solid line) electrodes. Inset plots show surfaces of the constant spinresolved local DOS evaluated at the Fermi energy. 


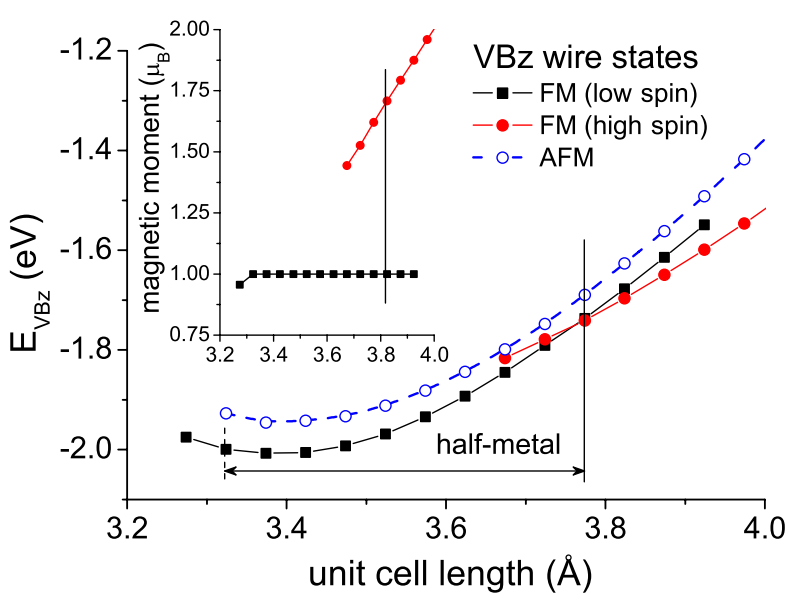

FIG. 5 (color online). Outer plot: Binding energy $E_{\mathrm{VBz}}$ between $\mathrm{V}$ and $\mathrm{Bz}$ as a function of the unit cell length. Black and red lines correspond to the low- and high-spin FM states, respectively. Blue line with open circles refers to the AFM state, which for any lattice constants is less preferable as compared with FM state. Inner plot: Behavior of the magnetic moment per unit cell under elongation of the wire.

action between V moments favors the FM phase. However, we cannot exclude another, indirect type of spin coupling, which is governed by the Kramers-Anderson mechanism $[29,30]$ of the superexchange interaction. For the considered system, because of the overlap of the HOMO (HOMO - 1) $\pi$-type orbital of benzene with the $d_{x z}, d_{y z}$ orbitals of vanadium, one of the $p$ electrons from benzene can hop over to one of the $\mathrm{V}$ atoms and the remaining unpaired $p$ electron on $\mathrm{Bz}$ can enter into a direct exchange with the other $\mathrm{V}$ atom. Applying the GoodenoughKanamori rules [30] regarding the sign of the effective exchange integral, we conclude that the indirect exchange interaction tends to align vanadium moments antiparallel and thus it competes with the direct coupling.

We have also studied the effect of the longitudinal stretching on the properties of the V-Bz wire. In Fig. 5 we show the binding energy between $\mathrm{V}$ and $\mathrm{Bz}$ as a function of the lattice constant together with the behavior of the magnetic moment (inset plot). We have found that a half-metallic state characterized by the integer magnetic moment is conserved up to $\sim 12 \%$, elongation of the wire. At the critical unit cell length of about $3.75 \AA$, the system exhibits a transition to a high-spin state. Just before the critical point the partially occupied minority spin $A_{1}$ band (b) nearby $E_{F}$ is transformed to the very narrow peak pinned to the Fermi level. Under further stretching this band is pushed above $E_{F}$ and a fractional charge of about $0.3 e$ per unit cell from the minority spin band flows to the upper lying majority spin band of $E_{1}$ symmetry (f) which now intersects the Fermi level close to the edge of the Brillouin zone. Thus, the transition is accompanied by an abrupt jump in the magnetic moment (Fig. 5, inset), from $1 \mu_{B}$ up to $\sim 1.6 \mu_{B}$ per unit cell, and the high-spin state becomes energetically favorable because of the gain in the exchange energy.

To summarize, the most important conclusion of our research is that recently synthesized multidecker vanadium-benzene clusters, when carrying spin-polarized currents, will work as highly effective spin filters.

We would like to thank B. Yavorsky, V. Stepanyuk, M. Ruben, and F. Weigend for fruitful discussions. This work was supported by DFG, No. SPP 1165.

[1] K. Miyajima et al., J. Am. Chem. Soc. 126, 13202 (2004).

[2] K. Miyajima et al., Eur. Phys. J. D 34, 177 (2005).

[3] K. Judai et al., Chem. Phys. Lett. 270, 23 (1997).

[4] K. Hoshino et al., J. Phys. Chem. 99, 3053 (1995).

[5] P. Weis et al., J. Phys. Chem. A 101, 8207 (1997).

[6] J. Wang et al., J. Am. Chem. Soc. 127, 2812 (2005).

[7] D. Rayane et al., Chem. Phys. Lett. 375, 506 (2003).

[8] R. A. de Groot et al., Phys. Rev. Lett. 50, 2024 (1983).

[9] An intriguing experiment would be a soft landing of $\mathrm{V}-\mathrm{Bz}$ clusters [K. Judai et al., Chem. Phys. Lett. 334, 277 (2001)] combined with a spin-polarized STM technique.

[10] V. R. Saunders, R. Dovesi, C. Roetti, R. Orlando, C. M. Zicovich-Wilson, N. M. Harrison, K. Doll, B. Civalleri, I. Bush, Ph. D'Arco, and M. Llunell, CRYSTAL 2003 User's Manual (University of Torino, Torino, 2003).

[11] J. P. Perdew and Y. Wang, Phys. Rev. B 45, 13244 (1992).

[12] R. Krishnan et al., J. Chem. Phys. 72, 650 (1980).

[13] W. J. Stevens et al., Can. J. Chem. 70, 612 (1992).

[14] T. Yasuike and S. Yabushita, J. Phys. Chem. A 103, 4533 (1999).

[15] R. Pandey et al., Chem. Phys. Lett. 321, 142 (2000).

[16] When this Letter was under review, closely related results on V-Bz wires were obtained by H. J. Xiang et al., J. Am. Chem. Soc. 128, 2310 (2006); Y. Mokrousov and N. Atodiresei (private communication).

[17] S. L. Dudarev et al., Phys. Rev. B 57, 1505 (1998).

[18] P.E. Blöchl, Phys. Rev. B 50, 17953 (1994).

[19] G. Kresse, J. Hafner, Phys. Rev. B 47, 558(R) (1993); G. Kresse and J. Furthmüller, Phys. Rev. B 54, 11169 (1996).

[20] A. D. Becke, J. Chem. Phys. 98, 5648 (1993).

[21] F. Geoffrey et al., Organometallics 2, 1150 (1983).

[22] M. P. Andrews et al., J. Phys. Chem. 90, 744 (1986).

[23] S. M. Mattara and R. Sammynaiken, J. Chem. Phys. 106, 1080 (1997).

[24] F. Mittendorfer and J. Hafner, Surf. Sci. 472, 133 (2001).

[25] M. Brandbyge, J.-L. Mozos, P. Ordejón, J. Taylor, and K. Stokbro, Phys. Rev. B 65, 165401 (2002).

[26] In both cases we used optimized basis sets of the double- $\zeta$ and even triple- $\zeta$ quality, including polarization functions.

[27] O. Treutler and R. Ahlrichs, J. Chem. Phys. 102, 346 (1995); K. Eichkorn, O. Treutler, H. Öhm, M. Häser, and R. Ahlrichs, Chem. Phys. Lett. 240, 283 (1995).

[28] F. Evers et al., Phys. Rev. B 69, 235411 (2004).

[29] P. W. Anderson, Phys. Rev. 79, 350 (1950).

[30] R. M. White, Quantum Theory of Magnetism (McGrawHill, New York, 1970). 